\title{
S Research Square \\ Long-Term Tolerability and Efficacy of
Carboplatin/Pemetrexed as Maintenance Therapy for a Patient With Lung Adenocarcinoma
}

Lixia Ju ( $\sim$ jvlixia@126.com )

Tongji University Affiliated Shanghai Pulmonary Hospital https://orcid.org/0000-0002-7158-9903 Juan Yang

Tongji University Affiliated Shanghai Pulmonary Hospital

\section{Case report}

Keywords: lung cancer, carboplatin, pemetrexed, maintenance therapy

Posted Date: May 27th, 2021

DOI: https://doi.org/10.21203/rs.3.rs-498643/v1

License: (c) (i) This work is licensed under a Creative Commons Attribution 4.0 International License.

Read Full License 


\section{Abstract}

\section{Background}

It is widely known that platinum-based doublet chemotherapy (PBC) only can be applied to first-line patients with non-small cell lung cancer (NSCLC) with good performance for 4-6 cycles. However, in this case report the patient has been treated with PBC for 30 cycles and more than three years.

\section{Case presentation}

A 63-year-old Chinese man was diagnosed with stage IVa lung adenocarcinoma, with Eastern Cooperative Oncology Group (ECOG) performance status (PS) of 0 . This patient did not respond to pemetrexed alone, but respond to pemetrexed and carboplatin, and once the chemotherapy was interrupted for more than two months, the disease would progressed. Moreover, there was little adverse reactions (AE), so we have treated him with $\mathrm{PBC}$ for 30 cycles and the progression-free survival (PFS) will be more than three years.

\section{Conclusions}

This is the first time to report PBC as maintenance therapy in patients with NSCLC. We hope that oncologists will notice that some diseases are very aggressive, and maintenance therapy are very important to some patients and may help to get longer overall survival time.

\section{Introduction}

In recent years, the treatment of NSCLC has undergone revolutionary progress. But according to the current guidelines, platinum-based chemotherapy (PBC) still represents a key first-line treatment for the patients with noncogene addicted NSCLC, and good PS and adequate organ function. Nevertheless, the period of PBC treatment is only 4-6 cycles, and then the patients only can wait for the advent of the progressed diseases. Although pemetrexed maintenance therapy significantly improved overall survival (OS) and PFS compared with placebo, and had a good safety profile in a phase 3 placebo-controlled study in patients with advanced NSCLC, the PFS and OS were only prolonged for 1.7 and 2.8 months ${ }^{[1]}$. Actually, it is far from meeting the clinical needs. We present the following case in accordance with the CARE reporting checklist.

\section{Case Presentation}

An 63-year-old Chinese man was admitted with a month history of dyspnea and swelling of the face and neck in May 2017. A chest computed tomography (CT) revealed an about $2.7 \mathrm{~cm}$ mass in the left upper lobe and enlarged left supraclavicular, mediastinal and hilar lymph nodes, and numbers of nodules distributed on two lungs (Fig. 1A). No other metastasis was observed by abdominal ultrasound examination, whole body bone scan and magnetic resonance imaging of the brain. The patient had no 
smoking history and family history of malignant tumor. Pathological examination of percutaneous fine needle biopsy of supraclavicular lymph nodes showed lung adenocarcinoma (Fig. 1B). The patient was clinically diagnosed with stage IVa lung adenocarcinoma, cT4N3M1a, with ECOG PS of 0.

The biopsied specimen from this patient was tested for epidermal growth factor receptor (EGFR), Kirsten rat sarcoma viral oncogene homolog (KRAS), and v-Raf murine sarcoma viral oncogene homolog $B$ (BRAF) using amplification-refractory mutation system (ARMS) assay with AmoyDx EGFR29 Mutations Detection Kit, AmoyDx KRAS19 Mutations Gene Detection Kit, AmoyDx BRAFV600E Mutation Gene Detection Kit, and was tested for anaplastic lymphoma kinase (ALK), The c-ros oncogene 1 (ROS1) status using reverse-transcriptase polymerase chain reaction (RT-PCR) assay with AmoyDx EML4-ALK Fusion Gene Detection Kit, AmoyDx ROS1 Fusion Gene Detection Kit (Amoy Diagnostics, Xiamen, China). The findings showed that the patient was negative for EGFR mutations, ALK/ROS1 fusions, and BRAF mutation, but was positive for KRAS mutation (Fig. 1C).

The patient was firstly treated with stent implantation in superior vena cava and carboplatin and pemetrexed chemotherapy in first-line setting. After two cycles of treatment, the disease was stable, but the mass and the lymph nodes were shrink. Then the patient was administered with another two cycles of chemotherapy and palliative thoracic radiotherapy. The disease was still stable with little shrink. Because of the advent of angiogenesis inhibitor, this patient was then treated with two cycles of bevacizumab and carboplatin/pemetrexed, and the disease partially responded (Fig. 1D). After 3 months of maintenance therapy with bevacizumab and pemetrexed, the disease was confirmed partial response (PR). Due to medical insurance restrictions of bevacizumab, it was stopped. The tumors were enlarged but not progressed after 2 cycles of maintenance therapy of pemetrexed. Then we tried to treat the patient with carboplatin and pemetrexed chemotherapy for two cycles, and the tumors were shrink. But once the chemotherapy was stopped for more than two months, the tumors were enlarged, so in the next two years, the patient has accepted for 30 cycles of carboplatin and pemetrexed chemotherapy and the disease is still stable. PFS isn't reached now, but it will be more than $\mathbf{4 0}$ months. Moreover, there was almost no adverse reaction (AE).

\section{Discussion}

According to the current guidelines, PBC only can be applied to first-line patients with NSCLC with good PS for 4-6 cycles. However, this patient in this paper did not respond to pemetrexed alone, but responded to carboplatin and pemetrexed, and once the chemotherapy was interrupted for more than two months, the disease would progress. Two phase III trials (JMEN and PARAMOUNT) have demonstrated a benefit from maintenance pemetrexed after platinum-based chemotherapy for advanced non-squamous NSCLC ${ }^{[2-4]}$. The most commonly AEs of chemotherapy are fatigue, anaemia, nausea, decreased appetite, leukocytopenia, thrombocytopenia, and vomiting, with most events being grade $1 / 2$ in severity ${ }^{[5,6]}$. There was no serious gastrointestinal or laboratory adverse reactions in this case. So we could treat him with doublet chemotherapy for nearly 30 cycles as maintaining therapy, and the PFS is more than three years. However, if the patient was switched to followup after 6 cycles of chemotherapy, the disease may have 
already progressed long ago, so it supported that maintenance therapy is very important for some patients.

\section{Declarations}

\section{Ethics approval and consent to participate}

Written informed consents were obtained from this patient.

\section{Consent for publication}

Not applicable.

\section{Availability of data and materials}

Not applicable.

\section{Funding}

Science and Technology Commission of Shanghai Municipality (17401932400; 19401930800); National Natural Science Foundation of China (81207106) 『Shanghai Pulmonary Hospital(fkgg1807)

\section{Competing interests}

The authors have no conflicts of interest to declare.

\section{Authors' contributions}

$\mathrm{JL}$ designed the study, analyzed the data and wrote the manuscript. Yang $\mathrm{J}$ corrected the data. All authors read and approved the final manuscript.

\section{Acknowledgements}

The authors would like to express their appreciation to the participant.

\section{References}

1. Ciuleanu T, Brodowicz T, Zielinski C, et al. Maintenance pemetrexed plus best supportive care versus placebo plus best supportive care for non-small-cell lung cancer: a randomised, double-blind, phase 3 study. Lancet 2009; 374: 1432-40.

2. Ciuleanu T, Brodowicz T, Zielinski $C$, et al. Maintenance pemetrexed plus best supportive care versus placebo plus best supportive care for non-small-cell lung cancer: a randomised, double-blind, phase 3 study. Lancet. 2009;374(9699):1432-1440. 
3. Paz-Ares L, de Marinis F, Dediu M, et al. Maintenance therapy with pemetrexed plus best supportive care versus placebo plus best supportive care after induction therapy with pemetrexed plus cisplatin for advanced non-squamous non-small-cell lung cancer (PARAMOUNT): a double-blind, phase 3, randomised controlled trial. The Lancet Oncology. 2012;13(3):247-255.

4. Paz-Ares LG, de Marinis F, Dediu M, et al. PARAMOUNT: Final overall survival results of the phase III study of maintenance pemetrexed versus placebo immediately after induction treatment with pemetrexed plus cisplatin for advanced nonsquamous non-small-cell lung cancer. JCO. 2013;31(23):2895-2902

5. Visser S, Koolen SLW, de Bruijn P, et al. Pemetrexed exposure predicts toxicity in advanced non-smallcell lung cancer: A prospective cohort study. Eur J Cancer. 2019,121:64-73

6. Langer CJ, Gadgeel SM, Borghaei H, Papadimitrakopoulou VA, Patnaik A, Powell SF, et al. Carboplatin and pemetrexed with or without pembrolizumab for advanced, non-squamous non-smallcell lung cancer: a randomised, phase 2 cohort of the open-label KEYNOTE-021 study. Lancet Oncol 2016;17:1497e508.

\section{Figures}


A
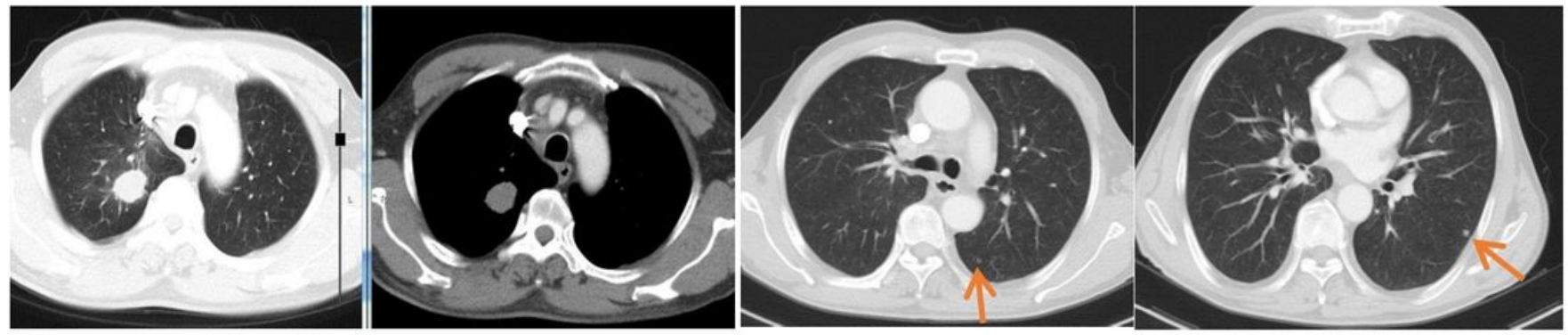

B
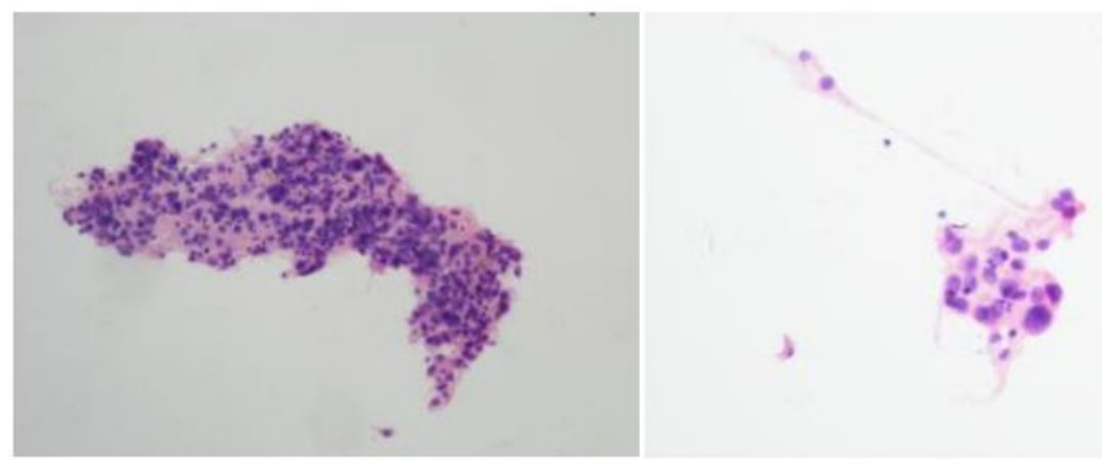

C

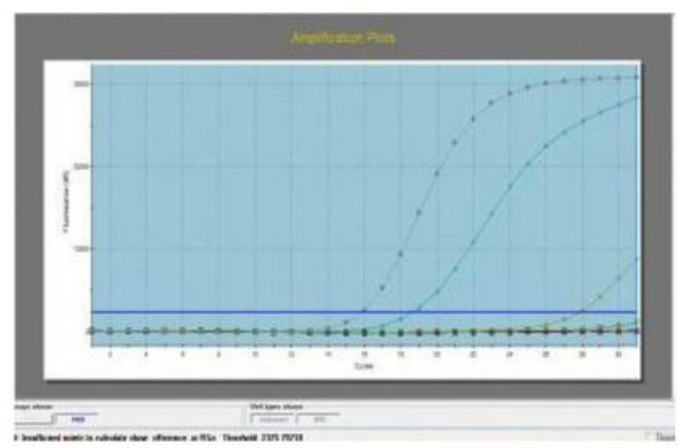

D

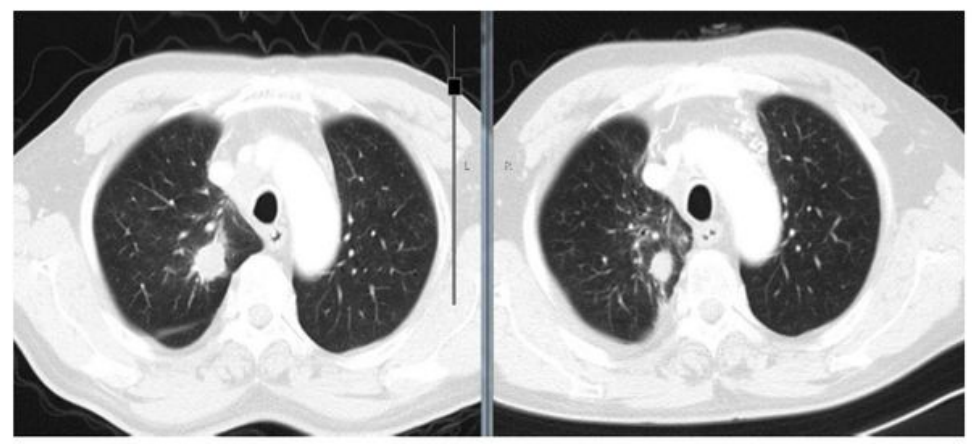

Figure 1

A: No other metastasis was observed by abdominal ultrasound examination, whole body bone scan and magnetic resonance imaging of the brain. B: The patient was clinically diagnosed with stage IVa lung adenocarcinoma, cT4N3M1a, with ECOG PS of 0 . C: The patient was firstly treated with stent implantation in superior vena cava and carboplatin and pemetrexed chemotherapy in first-line setting. After two cycles of treatment, the disease was stable, but the mass and the lymph nodes were shrink. D: After 3 months of maintenance therapy with bevacizumab and pemetrexed, the disease was confirmed partial response (PR). 